\title{
Singular values of multiplicative Toeplitz matrices
}

Article

Accepted Version

Hilberdink, T. (2017) Singular values of multiplicative Toeplitz matrices. Linear and Multilinear Algebra, 65 (4). pp. 813-829. ISSN 1563-5139 doi:

https://doi.org/10.1080/03081087.2016.1204978 Available at https://centaur.reading.ac.uk/66059/

It is advisable to refer to the publisher's version if you intend to cite from the work. See Guidance on citing.

To link to this article DOI: http://dx.doi.org/10.1080/03081087.2016.1204978

Publisher: Taylor \& Francis

All outputs in CentAUR are protected by Intellectual Property Rights law, including copyright law. Copyright and IPR is retained by the creators or other copyright holders. Terms and conditions for use of this material are defined in the End User Agreement.

\section{www.reading.ac.uk/centaur}

\section{CentAUR}

Central Archive at the University of Reading

Reading's research outputs online 


\title{
Singular Values of Multiplicative Toeplitz matrices
}

\author{
Titus Hilberdink \\ Department of Mathematics, University of Reading, Whiteknights, \\ PO Box 220, Reading RG6 6AX, UK; t.w.hilberdink@reading.ac.uk
}

\begin{abstract}
We study the asymptotic behaviour of the singular values of matrices with entries $a_{i j}=$ $f(i / j)$ if $j \mid i$ and zero otherwise, with $f$ an arithmetical function. In particular, we study the case where $f$ is multiplicative and $F(x):=\sum_{n \leq x}|f(n)|^{2}$ is regularly varying. Our main result is that, under quite general conditions, the singular values are, asymptotically, $\sqrt{\mu_{r} F(n)}$, where $\left\{\mu_{r}: r=1,2,3, \ldots\right\}$ are the eigenvalues of some positive Hilbert-Schmidt operator.
\end{abstract}

2010 AMS Mathematics Subject Classification: 11C20, 15A18.

Keywords and phrases: multiplicative functions, multiplicative Toeplitz matrices, singular values.

$\S 0$ Introduction - Multiplicative Toeplitz Matrices

In this article, we study the singular values of matrices of the form

$$
M_{n}(f) \stackrel{\text { def }}{=}\left(\begin{array}{cccccc}
f(1) & 0 & 0 & 0 & \cdots & 0 \\
f(2) & f(1) & 0 & 0 & \cdots & 0 \\
f(3) & 0 & f(1) & 0 & \cdots & 0 \\
f(4) & f(2) & 0 & f(1) & \cdots & 0 \\
\vdots & \vdots & \vdots & \vdots & \ddots & \vdots \\
f(n) & \cdot & \cdot & \cdot & \cdots & f(1)
\end{array}\right),
$$

where the $(i, j)^{\text {th }}$-entry is zero if $j$ does not divide $i$ and a function of $\frac{i}{j}$ if $j \mid i$. Such matrices are of number theoretic interest, in particular where the function $f(\cdot)$ has arithmetical properties. Their connection with multiplicative number theory derives from the simple observation that $M_{n}(f) M_{n}(g)=M_{n}(f * g)$, where $*$ denotes Dirichlet convolution; i.e. $(f * g)(n)=\sum_{d \mid n} f(d) g\left(\frac{n}{d}\right)$. As $*$ is a commutative operation, it follows that such matrices commute. Also $M_{n}(f)$ is invertible if and only if $f(1) \neq 0$, in which case $M_{n}(f)^{-1}=M_{n}\left(f^{-1}\right)$ where $f^{-1}$ is the Dirichlet inverse of $f$.

As an example, Redheffer's matrix [14], the determinant of which is related to the Riemann Hypothesis, involves the above matrix with $f \equiv 1$ (see also [12]). We are particularly interested in the behaviour of the singular values of $M_{n}(f)$ for large $n$; i.e. the square roots of the eigenvalues of $M_{n}(f)^{*} M_{n}(f)$, where $A^{*}$ denotes the conjugate tranpose of $A$. Various authors have studied eigenvalues of arithmetical matrices (see for example [11] and [13] to name just two, where the asymptotic behaviour of the largest/smallest eigenvalues are considered, and upper and lower bounds are given for other eigenvalues).

Matrices of the form (0.1) are a special case of matrices of the form

$$
\left(\begin{array}{ccccc}
a(1) & a\left(\frac{1}{2}\right) & a\left(\frac{1}{3}\right) & a\left(\frac{1}{4}\right) & \ldots \\
a(2) & a(1) & a\left(\frac{2}{3}\right) & a\left(\frac{1}{2}\right) & \ldots \\
a(3) & a\left(\frac{3}{2}\right) & a(1) & a\left(\frac{3}{4}\right) & \ldots \\
a(4) & a(2) & a\left(\frac{4}{3}\right) & a(1) & \ldots \\
\vdots & \vdots & \vdots & \vdots & \ddots
\end{array}\right)
$$


where the $(i, j)^{\text {th }}$-entry is a function of $i / j$. These (infinite) matrices $(0.2)$ are characterised by being constant along lines where $i / j$ is a given positive rational. In this sense they resemble Toeplitz matrices, which are constant on lines parallel to the diagonal; i.e. where $i-j$ is a given integer. For this reason these matrices were called multiplicative Toeplitz matrices (see [8]).

Toeplitz matrices are most naturally studied by associating with them a function (or 'symbol') whose Fourier coefficients make up the matrix (see for example, [3]). For matrices of the form $(0.2)$, one associates by analogy the series

$$
\sum_{q \in \mathbb{Q}^{+}} a(q) q^{i t},
$$

where $q$ ranges over the positive rationals. (In particular, matrices of the form (0.1) are associated with the Dirichlet series $\sum_{n=1}^{\infty} f(n) n^{i t}$.) If $\sum_{q \in \mathbb{Q}^{+}}|a(q)|$ converges, then the matrix (0.2) induces a bounded operator on $l^{2}$ (see [10]).

However, to fully exploit the connection between such matrices and their symbols requires that these functions are bounded and continuous (since almost periodic functions are bounded and continuous). This is turn requires that the $a(q)$ (hence also $f(n)$ ) must be small - indeed they must be square summable. In this paper we wish to deal with cases where $\sum|f(n)|^{2}$ diverges, and so our methods are quite different from those employed in [4], [9]. More precisely, we deal with the case where

$$
F(x) \stackrel{\text { def }}{=} \sum_{n \leq x}|f(n)|^{2}
$$

is regularly varying of index $\rho$ (see $\S 1.2$ for the definition). This notion occurs quite naturally here, as we show in the appendix.

The eigenvalues of $M_{n}(f)$ are uninteresting, all of them being $f(1)$. However, instead we consider its singular values. These are of number theoretic interest. For example, we shall see that

$$
\operatorname{tr}\left(M_{n}(f)^{*} M_{n}(f)\right)=\sum_{r=1}^{n} \sum_{d \mid r}|f(d)|^{2} .
$$

But the trace of a matrix is also the sum its eigenvalues, so this gives us new formulas for averages of arithmetical functions. Thus it is of interest to study these eigenvalues.

Our main result is that for $f$ multiplicative and $\rho>\frac{1}{2}$, the singular values of matrices of the form (0.1) behave like

$$
\sqrt{\mu_{r} F(n)}
$$

for large $n$, where $\mu_{1}, \mu_{2}, \ldots$ are the eigenvalues of some positive Hilbert-Schmidt operator. The completely multiplicative case is in some ways simpler, so we treat this case first in $\S 2$. The reason for the difference between the cases is that we need to relate sums like $\sum_{n \leq x} f(k n) f(l n)$ to $\sum_{n \leq x} f(n)^{2}$. For $f$ completely multiplicative this is easy, but for $f$ multiplicative we need to make some extra assumptions to obtain our result (in $\S 3$ ).

\section{$\S 1$. Preliminaries}

\subsection{Slow and regular variation}

We shall need the notion of regular variation (see [2]). A measurable function $f:(A, \infty) \rightarrow \mathbb{R}$, defined on some neighbourhood $(A, \infty)$ of $\infty$, is regularly varying of index $\rho$ if it is eventually positive and

$$
f(\lambda x) \sim \lambda^{\rho} f(x)
$$

as $x \rightarrow \infty$ for all $\lambda>0$. The set of regularly varying functions of index $\rho$ is denoted by $R_{\rho}$. A slowly varying function is a regularly varying function of index zero. 
Potter's bounds. If $f \in R_{\rho}$ and $f$ is bounded away from 0 and $\infty$ on compact subsets of $[1, \infty)$, then for every $\delta>0$ there exists $A$ such that

$$
\frac{f(y)}{f(x)} \leq A \max \left\{\left(\frac{y}{x}\right)^{\rho-\delta},\left(\frac{y}{x}\right)^{\rho+\delta}\right\} .
$$

for $x, y \geq 1$.

\subsection{Singular values; Trace Class and Hilbert-Schmidt operators}

Let $A$ be a compact operator on a Hilbert space $H$. Then $A^{*} A$ is a self-adjoint compact operator on $H$, whose spectrum, $\sigma\left(A^{*} A\right)$, lies in $[0, \infty)$. Furthermore, $\sigma\left(A^{*} A\right) \backslash\{0\}=\left\{\alpha_{n}: n \in \mathbb{N}\right\}$ for some decreasing sequence $\alpha_{n}$. The $n^{\text {th }}$ singular value ${ }^{1}$ of $A$ is $s_{n}(A)=\sqrt{\alpha_{n}}$. Then:

(i) $A$ is trace-class if $\sum_{n=1}^{\infty} s_{n}(A)$ converges;

(ii) $A$ is Hilbert-Schmidt if $\sum_{n=1}^{\infty} s_{n}(A)^{2}$ converges.

\section{Some relevant properties}

(a) The space of all trace-class operators is a Banach algebra with norm

$$
\|A\|_{1}=\sum_{n=1}^{\infty} s_{n}(A) .
$$

For $A$ trace-class, one can define $\operatorname{tr}(A)$, the trace of $A$, and $\operatorname{det}(I+A)$.

(b) The Hilbert-Schmidt operators on $H$ form a Hilbert space with inner product

$$
\langle A, B\rangle=\operatorname{tr}\left(A B^{*}\right),
$$

and corresponding norm

$$
\|A\|_{2}=\sqrt{\operatorname{tr}\left(A A^{*}\right)}=\sqrt{\sum_{n=1}^{\infty} s_{n}(A)^{2}} .
$$

We have $\|A\|=s_{1}(A)$, so $\|A\| \leq\|A\|_{2} \leq\|A\|_{1}$.

(c) If $A$ and $B$ are Hilbert-Schmidt, then $A B$ is trace-class, and $\|A B\|_{1} \leq\|A\|_{2}\|B\|_{2}$.

(d) Suppose $A$ has matrix representation $\left(a_{i j}\right)$ w.r.t. some orthonormal basis.

(i) If $\sum_{i, j \geq 1}\left|a_{i j}\right|$ converges, then $A$ is trace-class. Furthermore, if $A$ is self-adjoint and nonnegative, then $A$ is trace-class if and only if $\sum_{i=1}^{\infty} a_{i i}$ converges ([5], Chapter $1, \S 6$ Theorem $5)$.

(ii) If $A$ is trace-class, then with $\lambda_{n}$ denoting the eigenvalues of $A, \sum_{n}\left|\lambda_{n}\right| \leq \sum_{n} s_{n}(A)<\infty$, and the familiar formulae hold (irrespective of the basis):

$$
\operatorname{tr} A=\sum_{i=1}^{\infty} a_{i i}=\sum_{n=1}^{\infty} \lambda_{n} \quad \text { and } \quad \operatorname{det}(I+A)=\prod_{n=1}^{\infty}\left(1+\lambda_{n}\right) .
$$

\footnotetext{
${ }^{1}$ More generally, for a bounded linear operator $A$ on $H$, one defines

$$
s_{n}(A)=\inf \{\|A-F\|: \operatorname{rank} F<n\} .
$$
}

Then $A$ is compact if and only if $s_{n}(A) \rightarrow 0$ as $n \rightarrow \infty$ (see [7]). 
(iii) $A$ is Hilbert-Schmidt if and only if

$$
\sum_{i, j \geq 1}\left|a_{i j}\right|^{2}<\infty
$$

in which case the sum equals $\|A\|_{2}^{2}$ (again irrespective of the basis).

(e) Let $A, A_{n}$ be compact operators and suppose $A_{n} \rightarrow A$ in operator norm. Then $s_{r}\left(A_{n}\right) \rightarrow$ $s_{r}(A)$ for every $r \geq 1$.

This follows directly from the inequality $\left|s_{r}\left(A_{n}\right)-s_{r}(A)\right| \leq\left\|A_{n}-A\right\|$ (see [7], Chapter VI, Corollary 1.6). In particular, this applies to trace-class and Hilbert-Schmidt operators.

For these and more properties of these types of operators, see for example, [5], [6], [7].

\section{$\S 2$ Singular values of $M_{n}(f), f$ completely multiplicative}

The singular values of $M_{n}(f)$ are the square-roots of the eigenvalues of $M_{n}(f)^{*} M_{n}(f)$. More generally, consider $M_{n}(f)^{*} M_{n}(g)$. We have (with $[i, j]$ denoting the lcm of $i$ and $j$ )

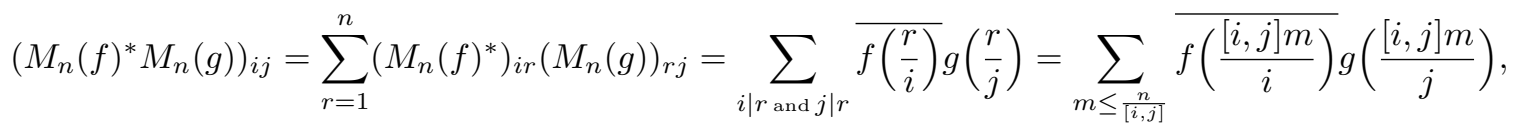

by putting $r=m[i, j]$. Using the fact that $[i, j](i, j)=i j$, we have proved:

\section{Proposition 2.1}

Let $f, g$ be arithmetical functions. Then

$$
\left(M_{n}(f)^{*} M_{n}(g)\right)_{i j}=\sum_{m \leq \frac{n}{[i, j]}} \overline{f\left(\frac{j m}{(i, j)}\right)} g\left(\frac{i m}{(i, j)}\right)
$$

In particular, $\left(M_{n}(f)^{*} M_{n}(g)\right)_{i i}=\sum_{m \leq \frac{n}{i}} \overline{f(m)} g(m)$, so that

$$
\operatorname{tr}\left(M_{n}(f)^{*} M_{n}(g)\right)=\sum_{i=1}^{n} \sum_{m \leq \frac{n}{i}} \overline{f(m)} g(m)=\sum_{i=1}^{n} \sum_{d \mid i} \overline{f(d)} g(d) .
$$

Notation For $f, g: \mathbb{N} \rightarrow \mathbb{C}$, let $F_{f, g}$ denote the function

$$
F_{f, g}(x)=\sum_{n \leq x} \overline{f(n)} g(n)
$$

In the case $f=g$, we write $F_{f}(x)$, or if no confusion is likely, just $F(x)$.

From Proposition 2.1, we see that $\left(M_{n}(f)^{*} M_{n}(g)\right)_{i i}=F_{f, g}\left(\frac{n}{i}\right)$. If $F_{f, g}(x) \rightarrow \infty$ as $x \rightarrow \infty$, then of course each of these entries also tends to infinity. However, if $F_{f, g}$ is regularly varying, say of index $\rho$, then

$$
\frac{F_{f, g}\left(\frac{n}{i}\right)}{F_{f, g}(n)} \rightarrow \frac{1}{i^{\rho}}
$$

The notion of regular variation is natural here, given we want some kind of convergence of the above form (see the appendix).

What about the behaviour for large $n$ of the other entries? In general this may be difficult to answer, but in the case that $f$ and $g$ are completely multiplicative, this can be done quite easily. For in this case, if $F_{f, g} \in R_{\rho}$, then

$$
\frac{1}{F_{f, g}(n)}\left(M_{n}(f)^{*} M_{n}(g)\right)_{i j}=\frac{\overline{f\left(\frac{j}{(i, j)}\right)} g\left(\frac{i}{(i, j)}\right) F_{f, g}\left(\frac{n}{[i, j]}\right)}{F_{f, g}(n)} \rightarrow \frac{\overline{f\left(\frac{j}{(i, j)}\right)} g\left(\frac{i}{(i, j)}\right)}{[i, j]^{\rho}}
$$


as $n \rightarrow \infty$; i.e. denoting the left-hand and right-hand sides by $e_{i j}^{(n)}$ and $e_{i j}$ respectively, we have $e_{i j}^{(n)} \rightarrow e_{i j}$. To be able to say something about the traces and determinants, we need to have a stronger form of convergence, namely norm convergence.

Let $E_{n}=\left(e_{i j}^{(n)}\right)_{i, j \leq n}$ and let $E$ denote the operator induced by the infinite matrix $\left(e_{i j}\right)_{i, j \geq 1}$.

\section{Theorem 2.2}

Let $f, g$ be two completely multiplicative functions, such that $F_{f, g}(\cdot)$ is regularly varying of index $\rho$ and bounded away from zero. Then

(a) $E$ is a Hilbert-Schmidt operator if and only if $\rho>\frac{1}{2}$ and $\sum_{n=1}^{\infty} \frac{|f(n)|^{2}+|g(n)|^{2}}{n^{2} \rho}$ converges.

(b) $E$ is trace-class if $\rho>1$ and $\sum_{n=1}^{\infty} \frac{|f(n)|+|g(n)|}{n^{\rho}}$ converges.

(c) If $\rho>\frac{1}{2}$ and $\sum_{n=1}^{\infty} \frac{|f(n)|^{2}+|g(n)|^{2}}{n^{2 \rho-\eta}}$ converges for some $\eta>0$, then $E_{n} \rightarrow E$ in Hilbert-Schmidt norm; i.e. $\left\|E_{n}-E\right\|_{2} \rightarrow 0$ as $n \rightarrow \infty$.

(d) If $\rho>1$ and $\sum_{n=1}^{\infty} \frac{|f(n)|+|g(n)|}{n^{\rho-\eta}}$ converges for some $\eta>0$, then $E_{n} \rightarrow E$ in trace-class norm; i.e. $\left\|E_{n}-E\right\|_{1} \rightarrow 0$ as $n \rightarrow \infty$.

To get information on the singular values of $M_{n}(f)$, take $g=f$, in which case $F_{f, g}$ becomes $F(x)=\sum_{n \leq x}|f(n)|^{2}$. Now, if $F(\cdot)$ is regularly varying of index $\rho$, then $\sum_{n=1}^{\infty}|f(n)|^{2} n^{-s}$ converges for $\Re s>\rho$, so that if $\rho>0$, the sum $\sum_{n=1}^{\infty}|f(n)|^{2} n^{\eta-2 \rho}$ converges for some $\eta>0$. This gives:

\section{Corollary 2.3}

Let $f$ be completely multiplicative, and suppose that $F(\cdot)$ is regularly varying of index $\rho$. Then

(a) $E_{n} \rightarrow E$ in Hilbert-Schmidt norm if and only if $\rho>\frac{1}{2}$;

(b) $E_{n} \rightarrow E$ in trace-class norm if and only if $\rho>1$.

Denote the singular values of $M_{n}(f)$ by $\lambda_{r, n}(r=1, \ldots, n)$ such that $\lambda_{1, n} \geq \lambda_{2, n} \geq \cdots \geq \lambda_{n, n}$, and let the eigenvalues of $E$ be $\left\{\mu_{r}\right\}_{r \geq 1}$ where $\mu_{1} \geq \mu_{2} \geq \cdots$. Then for $\rho>\frac{1}{2}$ and any fixed $r$,

$$
\lambda_{r, n}^{2} \sim \mu_{r} F(n) \quad \text { as } n \rightarrow \infty .
$$

Proof of Theorem 2.2. (a) By (d)(iii) from $\S 1.2, E$ is Hilbert-Schmidt if and only if

$$
\sum_{i, j \geq 1}\left|e_{i j}\right|^{2}<\infty
$$

But, on writing $(i, j)=d, i=m d, j=n d$ (so that $(m, n)=1$ ), this sum is

$$
\sum_{i, j \geq 1}\left|\frac{\overline{f\left(\frac{j}{(i, j)}\right)} g\left(\frac{i}{(i, j)}\right)}{[i, j]^{\rho}}\right|^{2}=\sum_{d=1}^{\infty} \sum_{\substack{m, n \geq 1 \\(m, n)=1}}\left|\frac{f(n) g(m)}{(n m d)^{\rho}}\right|^{2}=\zeta(2 \rho) \sum_{\substack{m, n \geq 1 \\(m, n)=1}} \frac{|f(n)|^{2}|g(m)|^{2}}{(n m)^{2 \rho}} .
$$

The RHS is less than $\zeta(2 \rho) \sum_{n=1}^{\infty} \frac{|f(n)|^{2}}{n^{2} \rho} \sum_{m=1}^{\infty} \frac{|g(m)|^{2}}{m^{2} \rho}$, but greater than both $\zeta(2 \rho) \sum_{n=1}^{\infty} \frac{|f(n)|^{2}}{n^{2 \rho}}$ and $\zeta(2 \rho) \sum_{n=1}^{\infty} \frac{|g(n)|^{2}}{n^{2 \rho}}$. Hence $E$ is Hilbert-Schmidt if and only if $\rho>\frac{1}{2}$ and both these sums are convergent.

(b) The proof that $E$ is trace-class if $\rho>1$ and both $\sum_{n=1}^{\infty} \frac{|f(n)|}{n^{\rho}}, \sum_{n=1}^{\infty} \frac{|g(n)|}{n^{\rho}}$ converge is identical to the above. In this case, we use the fact that $E$ is trace-class if $\sum_{i, j \geq 1}\left|e_{i j}\right|$ converges. 
(c) By the same methods as in (a) (with $\rho$ replaced by $\rho-\delta$ ), it follows from the extra assumptions that

$$
\sum_{i, j \geq 1}\left|e_{i j}\right|^{2}[i, j]^{\delta}
$$

converges for all $\delta>0$ sufficiently small.

We must show that

$$
\sum_{i, j \geq 1}\left|e_{i j}^{(n)}-e_{i j}\right|^{2} \rightarrow 0
$$

as $n \rightarrow \infty$. Now $e_{i j}^{(n)}=0$ for $[i, j]>n$, and $\sum_{[i, j]>n}\left|e_{i j}\right|^{2} \rightarrow 0$ with $n$ on account of the convergence of $\sum_{i, j \geq 1}\left|e_{i j}\right|^{2}$. Furthermore, for each $A$,

$$
\sum_{i, j \leq A}\left|e_{i j}^{(n)}-e_{i j}\right|^{2} \rightarrow 0
$$

as $n \rightarrow \infty$, since $e_{i j}^{(n)} \rightarrow e_{i j}$. There remains the range $[i, j] \leq n$ such that $\max \{i, j\}>A$.

Write $F_{f, g}(x)=x^{\rho} l(x)$ where $l(\cdot)$ is slowly-varying. Then $e_{i j}^{(n)}=e_{i j} l\left(\frac{n}{[i, j]}\right) / l(n)$. By Potter's bounds, given $\delta>0$, there exists $K>1$ such that

$$
\left|l\left(\frac{n}{[i, j]}\right) / l(n)\right| \leq K[i, j]^{\delta}
$$

whenever $[i, j] \leq n$. (For this we required to condition that $\left.F_{f, g}(\cdot)\right)$ be bounded away from 0 .)

Hence, taking $i, j$ over the remaining range, we have

$$
\begin{aligned}
\sum_{\max \{i, j\}>A}\left|e_{i j}^{(n)}-e_{i j}\right|^{2} & =\sum_{\max \{i, j\}>A}\left|e_{i j}\right|^{2}\left|1-l\left(\frac{n}{[i, j]}\right) / l(n)\right|^{2} \\
& \leq 2 \sum_{\max \{i, j\}>A}\left|e_{i j}\right|^{2}\left(1+\left|l\left(\frac{n}{[i, j]}\right) / l(n)\right|^{2}\right) \\
& \leq 4 K^{2} \sum_{\max \{i, j\}>A}\left|e_{i j}\right|^{2}[i, j]^{2 \delta} .
\end{aligned}
$$

But for all $\delta$ sufficiently small, the above sum converges. Hence we can choose $A$ so large that the RHS is less than any given $\varepsilon>0$. The result follows.

The proof for (d) is analogous, this time requiring $\sum_{i, j \geq 1}\left|e_{i j}^{(n)}-e_{i j}\right| \rightarrow 0$.

Proof of Corollary 2.3. Parts (a) and (b) follow immediately from Theorem 2.2 and, for (b) the reverse implication comes from 1.2(d)(i). For the final part, if $\rho>\frac{1}{2}$, then $E_{n} \rightarrow E$ in HilbertSchmidt norm. But the eigenvalues of $E_{n}$ are $\frac{\lambda_{r, n}^{2}}{F(n)}$ so by $1.2(\mathrm{e}), \frac{\lambda_{r, n}^{2}}{F(n)} \rightarrow \mu_{r}$ as $n \rightarrow \infty$.

\section{§3. The multiplicative case}

In the above we considered the case where $f$ (and $g$ ) is completely multiplicative. For our applications, we wish to generalize this to multiplicative functions. To obtain convergence of the entries as in Theorem 2.2 and Corollary 2.3, we require estimates for sums of the form

$$
\sum_{n \leq x} f(k n) g(l n)
$$


for $f, g$ multiplicative functions and $k, l$ coprime integers, given that we know the behaviour of the case $k=l=1$. As we are interested in the singular values of $M_{n}(f)$ we shall consider the special case when $g=\bar{f}$. The more general case can be treated similarly. Let

$$
F(x)=\sum_{n \leq x}|f(n)|^{2} \quad \text { and } \quad F_{k, l}(x)=\sum_{n \leq x} f(k n) \overline{f(l n)} .
$$

\section{Theorem 3.1}

Let $f$ be multiplicative, and suppose that $F(x)$ is regularly varying of index $\rho$. Let $k, l$ be coprime integers. Suppose that for every prime $p \mid k l$, the series $\sum_{m=0}^{\infty}\left|f\left(p^{m}\right)\right|^{2} p^{-m s}$ converges for $\Re s>\rho-\delta$ (some $\delta>0$ ) and is non-zero on the line $\Re s=\rho$. Then

$$
\frac{F_{k, l}(x)}{F(x)} \rightarrow \lambda(k) \overline{\lambda(l)}
$$

as $x \rightarrow \infty$ where, for $k=\prod_{p \mid k} p^{\alpha}$,

$$
\lambda(k) \stackrel{\text { def }}{=} \prod_{p \mid k}\left\{\frac{\sum_{m \geq 0} f\left(p^{m+\alpha}\right) \overline{f\left(p^{m}\right)} p^{-\rho m}}{\sum_{m \geq 0}\left|f\left(p^{m}\right)\right|^{2} p^{-\rho m}}\right\} .
$$

Proof. For $p \mid k l$, let

$$
h_{p}(z)=\sum_{m=0}^{\infty}\left|f\left(p^{m}\right)\right|^{2} z^{m}
$$

The assumptions imply that this power series has a radius of convergence larger than $p^{-\rho}$ and that $h_{p}(z) \neq 0$ for $|z|=p^{-\rho}$. This in turn implies there exists $\eta>0$ such that $h_{p}(z) \neq 0$ in the annulus

$$
A=\left\{z \in \mathbb{C}: p^{-\rho}-\eta<|z|<p^{-\rho}+\eta\right\} .
$$

The power series

$$
\sum_{m=0}^{\infty} f\left(p^{m+r}\right) \overline{f\left(p^{m}\right)} z^{m}
$$

has a radius of convergence at least as large as that for $h_{p}(z)$, and hence converges for $|z|<p^{-\rho}+\eta$. Thus $\left(1 / h_{p}(z)\right) \sum_{m=0}^{\infty} f\left(p^{m+r}\right) \overline{f\left(p^{m}\right)} z^{m}$ is analytic in $A$ and has a Laurent series:

$$
\frac{1}{h_{p}(z)} \sum_{m=0}^{\infty} f\left(p^{m+r}\right) \overline{f\left(p^{m}\right)} z^{m}=\sum_{n=-\infty}^{\infty} \alpha_{n} z^{n} .
$$

Replacing $z$ by $p^{-s}$, this implies that there exists $\eta^{\prime}>0$ such that

$$
\frac{1}{h_{p}\left(p^{-s}\right)} \sum_{m=0}^{\infty} \frac{f\left(p^{m+r}\right) \overline{f\left(p^{m}\right)}}{p^{m s}}=\sum_{n=-\infty}^{\infty} \frac{\alpha_{n}}{p^{n s}} \quad \text { for } \rho-\eta^{\prime}<\Re s<\rho+\eta^{\prime} .
$$

Similarly for $\frac{1}{h_{p}\left(p^{-s}\right)} \sum_{m=0}^{\infty} f\left(p^{m}\right) \overline{f\left(p^{m+r}\right)} p^{-m s}$. Let

$$
\mathcal{F}(s)=\sum_{n=1}^{\infty} \frac{|f(n)|^{2}}{n^{s}} \quad \text { and } \quad \mathcal{F}_{k, l}(s)=\sum_{n=1}^{\infty} \frac{f(k n) \overline{f(l n)}}{n^{s}} .
$$

These series are absolutely convergent for $\Re s>\rho$ since

$$
\left|F_{k, l}(x)\right| \leq\left(\sum_{n \leq x}|f(k n)|^{2} \sum_{n \leq x}|f(l n)|^{2}\right)^{1 / 2} \leq \sqrt{F(k x) F(l x)} \sim(k l)^{\rho / 2} F(x) .
$$


As $f(n)$ is multiplicative, we have the Euler products

$$
\mathcal{F}(s)=\prod_{p} \sum_{m=0}^{\infty} \frac{\left|f\left(p^{m}\right)\right|^{2}}{p^{m s}} \quad \text { and } \quad \mathcal{F}_{k, l}(s)=\prod_{p} \sum_{m=0}^{\infty} \frac{f\left(p^{m+\alpha}\right) \overline{f\left(p^{m+\beta}\right)}}{p^{m s}} .
$$

(Recall $k=\prod_{p \mid k} p^{\alpha}$ and $l=\prod_{p \mid l} p^{\beta}$.) If $p \nmid k l$ then $\alpha=\beta=0$, and $f\left(p^{m+\alpha}\right) \overline{f\left(p^{m+\beta}\right)}$ becomes $\left|f\left(p^{m}\right)\right|^{2}$. Splitting the product for $\mathcal{F}_{k, l}(s)$ into those primes for which $p$ divides $k l$ and those that do not, and cancelling the $p \nmid k l$ terms with those in the denominator gives

$$
\mathcal{F}_{k, l}(s)=\prod_{p \mid k l} \sum_{m=0}^{\infty} f\left(p^{m+\alpha}\right) \overline{f\left(p^{m+\beta}\right)} p^{-m s} \cdot \prod_{p \nmid k l} \sum_{m=0}^{\infty}\left|f\left(p^{m}\right)\right|^{2} p^{-m s} .
$$

But if $p \mid k$ then $\beta=0$, while if $p \mid l$ then $\alpha=0$, since $(k, l)=1$. Hence

$$
\mathcal{F}_{k, l}(s)=\prod_{p \mid k} \frac{\sum_{m=0}^{\infty} f\left(p^{m+\alpha}\right) \overline{f\left(p^{m}\right)} p^{-m s}}{\sum_{m=0}^{\infty}\left|f\left(p^{m}\right)\right|^{2} p^{-m s}} \prod_{p \mid l} \frac{\sum_{m=0}^{\infty} f\left(p^{m}\right) \overline{f\left(p^{m+\beta}\right)} p^{-m s}}{\sum_{m=0}^{\infty}\left|f\left(p^{m}\right)\right|^{2} p^{-m s}} \mathcal{F}(s),
$$

for $\rho<\Re s<\rho+\eta^{\prime}$. But each term in the product (with $p \mid k$ and $p \mid l$ respectively) is a series of the form $\sum_{-\infty}^{\infty} \beta_{n} p^{-n s}$ as in (3.1). Now for $n=p_{1}^{a_{1}} \ldots p_{m}^{a_{m}}$ (with $a_{i} \geq 1$ ) let

$$
S_{n}=\left\{p_{1}^{k_{1}} \ldots p_{m}^{k_{m}}: k_{1}, \ldots, k_{m} \in \mathbb{Z}\right\} .
$$

Thus

$$
\prod_{p \mid k} \frac{\sum_{m=0}^{\infty} f\left(p^{m+\alpha}\right) \overline{f\left(p^{m}\right)} p^{-m s}}{\sum_{m=0}^{\infty}\left|f\left(p^{m}\right)\right|^{2} p^{-m s}} \prod_{p \mid l} \frac{\sum_{m=0}^{\infty} f\left(p^{m}\right) \overline{f\left(p^{m+\beta}\right)} p^{-m s}}{\sum_{m=0}^{\infty}\left|f\left(p^{m}\right)\right|^{2} p^{-m s}}=\sum_{q \in S_{k l}} \frac{\gamma_{q}}{q^{s}},
$$

for some coefficients $\gamma_{q}$, where the series converges absolutely for $\rho-\eta^{\prime \prime}<\Re s<\rho+\eta^{\prime \prime}$, some $\eta^{\prime \prime}>0$. Note that from (3.2) we have

$$
\sum_{q \in S_{k l}} \frac{\gamma_{q}}{q^{\rho}}=\lambda(k) \overline{\lambda(l)}
$$

Let

$$
A(x)=\sum_{\substack{q \in S_{k l} \\ q \leq x}} \gamma_{q}
$$

From (3.2) it follows that for $s \in\left(\rho-\eta^{\prime \prime}, \rho+\eta^{\prime \prime}\right)$, we have $|A(x)| \leq x^{s} \sum_{q \leq x}\left|\gamma_{q}\right| q^{-s}=O\left(x^{s}\right)$. Hence for some $\delta^{\prime}>0$,

$$
A(x)= \begin{cases}O\left(x^{\rho+\delta^{\prime}}\right) & \text { for } x \leq 1 \\ O\left(x^{\rho-\delta^{\prime}}\right) & \text { for } x \geq 1\end{cases}
$$

From above

$$
\mathcal{F}_{k, l}(s)=\left(\sum_{q \in S_{k l}} \frac{\gamma_{q}}{q^{s}}\right) \mathcal{F}(s),
$$

for $\rho<\Re s<\rho+\eta^{\prime \prime}$. Fix $c \in\left(\rho, \rho+\eta^{\prime}\right)$. If we ignore questions of convergence we should have

$$
\frac{1}{2 \pi i} \int_{(c)} \frac{\mathcal{F}_{k, l}(s)}{s} x^{s} d s=\frac{1}{2 \pi i} \int_{(c)}\left(\sum_{q \in S_{k l}} \frac{\gamma_{q}}{q^{s}}\right) \frac{\mathcal{F}(s)}{s} x^{s} d s=\sum_{q \in S_{k l}} \gamma_{q}\left(\frac{1}{2 \pi i} \int_{(c)} \frac{\mathcal{F}(s)}{s}\left(\frac{x}{q}\right)^{s} d s\right) .
$$

However, interchanging the sum and integral cannot be justified (at least easily) since, although the series converges uniformly and boundedly, the integral does not converge absolutely. To get round this let

$$
G(x)=\int_{0}^{x} F(t) d t \quad \text { and } \quad G_{k, l}(x)=\int_{0}^{x} F_{k, l}(t) d t
$$


Note that since $F(x)=F([x])$ and $F_{k, l}(x)=F_{k, l}([x])$, we have

$$
\frac{G(x+\delta)-G(x)}{\delta}=F(x) \quad \text { and } \quad \frac{G_{k, l}(x+\delta)-G_{k, l}(x)}{\delta}=F_{k, l}(x)
$$

for all $\delta>0$ sufficiently small. Also we have the Mellin transforms

$$
G(x)=\frac{1}{2 \pi i} \int_{(c)} \frac{\mathcal{F}(s)}{s(s+1)} x^{s+1} d s \quad \text { and } \quad G_{k, l}(x)=\frac{1}{2 \pi i} \int_{(c)} \frac{\mathcal{F}_{k, l}(s)}{s(s+1)} x^{s+1} d s
$$

for every $c>1$ and $x>0$. These integrals have the advantage that they converge absolutely. Thus by uniform and absolute convergence we can interchange sum and integral to give

$$
G_{k, l}(x)=\frac{1}{2 \pi i} \int_{(c)} \frac{\mathcal{F}_{k, l}(s)}{s(s+1)} x^{s+1} d s=\frac{1}{2 \pi i} \int_{(c)}\left(\sum_{q \in S_{k l}} \frac{\gamma_{q}}{q^{s}}\right) \frac{\mathcal{F}(s)}{s(s+1)} x^{s+1} d s=\sum_{q \in S_{k l}} q \gamma_{q} G\left(\frac{x}{q}\right) .
$$

Writing $F^{*}(x)$ for $\sum_{n \leq x}^{\prime} f(n)$ where the $/$ indicates the last term is to be halved if $x \in \mathbb{N}$ and $F_{k, l}^{*}(x)$ analogously, this gives

$$
F_{k, l}^{*}(x)=\sum_{q \in S_{k l}} \gamma_{q} F^{*}\left(\frac{x}{q}\right)
$$

From this we can deduce that

$$
F_{k, l}(x)=\sum_{q \in S_{k l}} \gamma_{q} F\left(\frac{x}{q}\right) .
$$

To see this, first note that the righthand side of (3.3) converges absolutely: by Potter's bounds,

$$
\frac{F(x / q)}{F(x)} \leq A \max \left\{q^{-\rho-\delta}, q^{-\rho+\delta}\right\},
$$

for $x, q \geq 1$ and any given $\delta>0$ and some $A=A(\delta)$, while $\sum_{q}\left|\gamma_{q}\right| / q^{\rho-\eta}$ converges for $|\eta|$ sufficiently small.

Now for $q=\frac{m}{n}$ with $m, n$ coprime, write $\|q\|=\max \{m, n\}$. Let $\delta \in(0,1)$. We have

$$
\frac{G_{k, l}(x+\delta)-G_{k, l}(x)}{\delta}=\sum_{q \in S_{k l}} \gamma_{q}\left(\frac{G((x+\delta) / q)-G(x / q)}{\delta / q}\right) .
$$

Let $\delta \rightarrow 0$. For every $N$,

$$
\sum_{\substack{q \in S_{k l} \\\|q\| \leq N}} \gamma_{q}\left(\frac{G((x+\delta) / q)-G(x / q)}{\delta / q}\right) \rightarrow \sum_{\substack{q \in S_{k l} \\\|q\| \leq N}} \gamma_{q} F\left(\frac{x}{q}\right) .
$$

For $\|q\|>N$, using $G(a+b)-G(a) \leq b F(a+b)$ for $a>0$, we have

$$
\left|\sum_{\substack{q \in S_{k l} \\\|q\|>N}} \gamma_{q}\left(\frac{G((x+\delta) / q)-G(x / q)}{\delta / q}\right)\right| \leq \sum_{\substack{q \in S_{k l} \\\|q\|>N}}\left|\gamma_{q}\right| F\left(\frac{x+1}{q}\right) .
$$

This is independent of $\delta$. By the absolute convergence of the series it tends to 0 as $N \rightarrow \infty$. Hence (3.3) follows. But

$$
\begin{aligned}
\sum_{q \in S_{k l}} \gamma_{q} F\left(\frac{x}{q}\right) & =\sum_{q \in S_{k l}} \gamma_{q} \sum_{n \leq x / q} f(n)=\sum_{\substack{n \in \mathbb{N}, q \in S_{k l} \\
n q \leq x}} \gamma_{q} f(n) \\
& =\sum_{n=1}^{\infty} f(n) \sum_{\substack{q \in S_{k l} \\
q \leq x / n}} \gamma_{q}=\sum_{n=1}^{\infty} f(n) A\left(\frac{x}{n}\right) .
\end{aligned}
$$


The right-hand side of (3.4) is just a Mellin-Stieltjes transform; i.e. $F_{k, l}(x)=\int_{0}^{\infty} A(x / t) d F(t)$. The conditions of Theorem 4.4.2 from [2] (with $U=F$ and $k=A$ ) are satisfied and can be applied to give

$$
F_{k, l}(x) \sim\left(\rho \int_{0}^{\infty} \frac{A(t)}{t^{\rho+1}} d t\right) F(x) .
$$

But

$$
\begin{aligned}
\rho \int_{0}^{\infty} \frac{A(t)}{t^{\rho+1}} d t & =\lim _{x \rightarrow \infty, y \rightarrow 0+}\left(\frac{A(y)}{y^{\rho}}-\frac{A(x)}{x^{\rho}}+\int_{y}^{x} \frac{1}{t^{\rho}} d A(t)\right)= \\
& =\int_{0}^{\infty} \frac{1}{t^{\rho}} d A(t)=\sum_{q \in S_{k l}} \frac{\gamma_{q}}{q^{\rho}}=\lambda(k) \overline{\lambda(l)} .
\end{aligned}
$$

Remarks. (i) The condition that $\sum_{m=0}^{\infty}\left|f\left(p^{m}\right)\right|{ }^{2} p^{-m s}$ be non-zero on the line $\Re s=\rho$ is sufficient for the limit to exist, but not necessary. For example, let $f$ be the multiplicative function

$$
f(n)=\left\{\begin{array}{cl}
1 & \text { if } n \text { is odd } \\
\sqrt{2} & \text { if } n=2 m \text { with } m \text { odd } . \\
0 & \text { if } 4 \mid n
\end{array}\right.
$$

Then $F(x)=\sum_{n \leq x}|f(n)|^{2}=x+O(1)$ which is regularly varying of index 1, and an easy calculation shows that $F_{k, l}(x) / F(x) \rightarrow \mu_{k, l}$ where

$$
\mu_{k, l}=\left\{\begin{array}{ll}
1 & \text { if } k, l \text { both odd } \\
\frac{1}{\sqrt{2}} & \text { if } k=2 m \text { with } m \text { odd and } l \text { odd, or vice versa } \\
1 & \text { if } k, l=2 m, 2 n \text { with } m, n \text { odd } \\
0 & \text { if } 4 \mid k \text { or } 4 \mid l
\end{array} .\right.
$$

But $\sum_{m=0}^{\infty}\left|f\left(2^{m}\right)\right|^{2} 2^{-m s}=1+2^{1-s}$ which has zeros on the line $\Re s=1$.

(ii) The condition that $\sum_{m=0}^{\infty}\left|f\left(p^{m}\right)\right|^{2} p^{-m(\rho-\delta)}$ converges for some $\delta>0$ is also not necessary, even with $\delta=0$. For example, let $f$ be the (non-completely) multiplicative function

$$
f(n)=f\left(2^{m} r\right)=2^{m / 2}(m+1)
$$

if $n=2^{m} r$ with $m \geq 0$ and $r$ odd. Then (with $m \geq 0$ and $r$ odd)

$$
F(x)=\sum_{2^{m} r \leq x}\left|f\left(2^{m} r\right)\right|^{2}=\sum_{2^{m} \leq x} 2^{m}(m+1)^{2}\left(\frac{x}{2^{m+1}}+O(1)\right) \sim \frac{x(\log x)^{3}}{6(\log 2)^{3}},
$$

which is regularly varying of index 1 . An easy calculation shows that

$$
\frac{F_{k, l}(x)}{F(x)} \rightarrow 2^{\frac{1}{2}(m+n)}
$$

for $k=2^{m} r$ and $l=2^{n} s$ with $r, s$ odd. But $\sum_{m=0}^{\infty}\left|f\left(2^{m}\right)\right|^{2} 2^{-m}$ diverges.

It is conceivable that no extra condition is required as in the completely multiplicative case, but this is apparently not easy to prove - if indeed it is true.

(iii) It would be nice to use a Tauberian theorem directly, say Theorem 4.9.1 from [2], but this requires a continuous kernel $k$. 
Now we apply this to generalize Corollary 2.3 to multiplicative $f$. Let $E_{n}=\left(e_{i j}^{(n)}\right)_{i, j \leq n}$ and $E=\left(e_{i j}\right)_{i, j \geq 1}$ where

$$
e_{i j}^{(n)}=\frac{\left(M_{n}(f)^{*} M_{n}(f)\right)_{i j}}{F(n)} \quad \text { and } \quad e_{i j}=\lambda\left(\frac{i}{(i, j)}\right) \overline{\lambda\left(\frac{j}{(i, j)}\right)}[i, j]^{-\rho},
$$

and $\lambda(\cdot)$ is as in Theorem 3.1. Note that with $k=\frac{i}{(i, j)}$ and $l=\frac{j}{(i, j)}($ so $(k, l)=1)$, we have

$$
e_{i j}^{(n)}=\frac{F_{k, l}\left(\frac{n}{[i, j]}\right)}{F(n)} \rightarrow e_{i j},
$$

for every $i, j \geq 1$, under the conditions of Theorem 3.1.

\section{Theorem 3.2}

Let $f$ be multiplicative, and suppose that $F(x)$ is regularly varying of index $\rho$. Suppose further that for every prime $p$, the series $\sum_{m=0}^{\infty}\left|f\left(p^{m}\right)\right|^{2} p^{-m s}$, converges for $\Re s>\rho-\delta$ (some $\delta>0$, possibly depending on $p)$, and is non-zero for $\Re s=\rho$. Then

(a) $E$ is trace-class if and only if $\rho>1$;

(b) $E$ is Hilbert-Schmidt if and only if $\rho>\frac{1}{2}$ and $\sum_{p} \frac{|\lambda(p)|^{2}}{p^{2 \rho}}$ converges.

Furthermore, suppose $\rho>\frac{1}{2}, \sum_{n=1}^{\infty} \frac{|\lambda(n)|^{2}}{n^{2 \rho-\eta}}$ converges for some $\eta>0$, and for all $\varepsilon>0$, $F_{k, l}(x) \ll_{\varepsilon}(k l)^{\varepsilon}|\lambda(k l)| F(x)$ whenever $(k, l)=1$. Then $E_{n} \rightarrow E$ in Hilbert-Schmidt norm.

Consequently, with $\lambda_{r, n}$ denoting the $r^{\text {th }}$ singular value of $M_{n}(f)$ and $\mu_{r}$ the $r^{\text {th }}$ eigenvalue of $E$, we have

$$
\lambda_{r, n}^{2} \sim \mu_{r} F(n) .
$$

Proof. (a) As $E$ is self-adjoint, it is trace class if and only if $\sum_{i} e_{i i}$ converges, by $1.2 \mathrm{~d}(\mathrm{i})$. But $e_{i i}=i^{-\rho}$, so the condition holds if and only if $\rho>1$.

(b) $E$ is Hilbert-Schmidt if and only if $\sum_{i, j \geq 1}\left|e_{i j}\right|^{2}$ converges. But this sum is

$$
\sum_{i, j \geq 1}\left|\lambda\left(\frac{i}{(i, j)}\right) \overline{\lambda\left(\frac{j}{(i, j)}\right)}[i, j]^{-\rho}\right|^{2}=\sum_{d=1}^{\infty} \sum_{\substack{m, n \geq 1 \\(m, n)=1}}\left|\frac{\lambda(n) \overline{\lambda(m)}}{(n m d)^{\rho}}\right|^{2}=\zeta(2 \rho) \sum_{\substack{m, n \geq 1 \\(m, n)=1}} \frac{|\lambda(n)|^{2}|\lambda(m)|^{2}}{(n m)^{2 \rho}} .
$$

The RHS is less than $\zeta(2 \rho)\left(\sum_{n=1}^{\infty} \frac{|\lambda(n)|^{2}}{n^{2 \rho}}\right)^{2}$ but greater than $\zeta(2 \rho) \sum_{n=1}^{\infty} \frac{|\lambda(n)|^{2}}{n^{2 \rho}}$. Hence $E$ is Hilbert-Schmidt if and only if $\rho>\frac{1}{2}$ and $\sum_{n=1}^{\infty} \frac{|\lambda(n)|^{2}}{n^{2 \rho}}$ converges. But $\lambda(\cdot)$ is multiplicative, so this is equivalent to the convergence of

$$
\sum_{p} \sum_{k=1}^{\infty} \frac{\left|\lambda\left(p^{k}\right)\right|^{2}}{p^{2 k \rho}}
$$

However,

$$
\left|\lambda\left(p^{k}\right)\right|^{2}=\frac{\left|\sum_{m=0}^{\infty} f\left(p^{m+k}\right) \overline{f\left(p^{m}\right)} p^{-\rho m}\right|^{2}}{\left.\left.\left|\sum_{m=0}^{\infty}\right| f\left(p^{m}\right)\right|^{2} p^{-\rho m}\right|^{2}} \leq \frac{\sum_{m=0}^{\infty}\left|f\left(p^{m+k}\right)\right|^{2} p^{-\rho m}}{\sum_{m=0}^{\infty}\left|f\left(p^{m}\right)\right|^{2} p^{-\rho m}} \leq p^{\rho k},
$$

and therefore $\sum_{p, k \geq 2}\left|\lambda\left(p^{k}\right)\right|^{2} p^{-2 k \rho}$ converges. It follows that $E$ is Hilbert-Schmidt if and only if $\rho>\frac{1}{2}$ and $\sum_{p}|\lambda(p)|^{2} p^{-2 \rho}$ converges. 
For the final part, if $\rho>\frac{1}{2}$ and $\sum_{n=1}^{\infty} \frac{|\lambda(n)|^{2}}{n^{2} \rho-\eta}$ converges for some $\eta>0$ then, as in the proof of Theorem 2.2,

$$
\sum_{i, j \geq 1}\left|e_{i j}\right|^{2}[i, j]^{\eta} \quad \text { converges for some (possibly different) } \eta>0 \text {. }
$$

We must show that $\sum_{i, j \geq 1}\left|e_{i j}^{(n)}-e_{i j}\right|^{2} \rightarrow 0$ as $n \rightarrow \infty$. Again, for each $A, \sum_{i, j \leq A}\left|e_{i j}^{(n)}-e_{i j}\right|^{2} \rightarrow 0$ as $n \rightarrow \infty$, since $e_{i j}^{(n)} \rightarrow e_{i j}$. Further, the convergence of $\sum_{i, j \geq 1}\left|e_{i j}\right|^{2}$ implies

$$
\sum_{\max \{i, j\}>A}\left|e_{i j}\right|^{2}<\varepsilon(A)
$$

where $\varepsilon(A) \rightarrow 0$ as $A \rightarrow \infty$. There remains the sum

$$
\sum_{\max \{i, j\}>A}\left|e_{i j}^{(n)}\right|^{2}
$$

But, with $k=\frac{i}{(i, j)}$ and $l=\frac{j}{(i, j)}$, we have

$$
\left|e_{i j}^{(n)}\right|=\frac{\left|F_{k, l}\left(\frac{n}{[i, j]}\right)\right|}{F(n)}=\frac{\left|F_{k, l}\left(\frac{n}{[i, j]}\right)\right|}{F\left(\frac{n}{[i, j]}\right)} \cdot \frac{F\left(\frac{n}{[i, j]}\right)}{F(n)} \stackrel{(i)}{\leq} A_{\delta, \varepsilon} \frac{(k l)^{\varepsilon}|\lambda(k l)|}{[i, j]^{\rho-\delta}} \stackrel{(i i)}{\leq} A_{\delta, \varepsilon}[i, j]^{\delta+\varepsilon}\left|e_{i j}\right|
$$

for all $\delta, \varepsilon>0$ and some constant $A_{\delta, \varepsilon}$ (i) by the assumption and Potter's bounds and (ii) since $k l \leq[i, j]$. Choosing $\delta$ and $\varepsilon$ sufficiently small so that $\delta+\varepsilon<\eta / 2$, we see that $\sum_{\max \{i, j\}>A}\left|e_{i j}^{(n)}\right|^{2}<$ $\varepsilon(A) \rightarrow 0$ as $A \rightarrow \infty$. The result follows.

\section{§4. Some examples}

(a) In [10], the largest singular value of $M_{n}(f)$ was discussed and its close connection to the largest eigenvalue of the $n \times n$ matrix $\left(f\left(\frac{i j}{(i, j)^{2}}\right)\right)_{i, j \leq n}$ in case $f$ is completely multiplicative.

The particular case $f(n)=n^{-\alpha}$ was dealt with in [9] with estimates obtained for the various ranges of $\alpha$. Denoting the largest singular value of $M_{n}(f)$ by $\lambda_{1, n}$ (denoted by $B_{\alpha}(n)$ in [9]), it was shown that for $\alpha<\frac{1}{2}$ one has

$$
\lambda_{1, n} \asymp n^{\frac{1}{2}-\alpha} .
$$

Note that in this case, $F(x)=\sum_{n \leq x}|f(n)|^{2} \sim \frac{x^{1-2 \alpha}}{1-2 \alpha}$ which is regularly varying of index $\rho=1-2 \alpha$. Observe that $\rho>\frac{1}{2}$ when $\alpha<\frac{1}{4}$. As such, we can apply Corollary 2.3. Thus

$$
\lambda_{1, n} \sim \sqrt{\frac{\mu_{1}(\alpha)}{1-2 \alpha}} n^{\frac{1}{2}-\alpha} \quad\left(\alpha<\frac{1}{4}\right),
$$

where $\mu_{1}(\alpha)$ is the largest eigenvalue of the Hilbert-Schmidt operator $E_{\alpha}=\left((i j)^{\alpha} /[i, j]\right)_{i, j \geq 1}$. Furthermore, the $r^{\text {th }}$ singular value of $M_{n}(f)$, say $\lambda_{r, n}$ satisfies

$$
\lambda_{r, n} \sim \sqrt{\frac{\mu_{r}(\alpha)}{1-2 \alpha}} n^{\frac{1}{2}-\alpha} \quad \text { as } n \rightarrow \infty
$$

where $\mu_{r}(\alpha)$ is the $r^{\text {th }}$ eigenvalue of $E_{\alpha}$.

The special case $\alpha=0$ was discussed recently in [13] and [1]. Mattila and Haukkanen [13] conjectured and Altinişik and Büyükköse [1] proved that $\lambda_{1, n}$ increases with $n$, and further 
proved that $\lambda_{1, n}^{2} \geq n$. The above tells us that $\lambda_{1, n}^{2} \sim \mu_{1} n$ and more generally that $\lambda_{r, n}^{2} \sim \mu_{r} n$ where $\mu_{r}$ is the $r^{\text {th }}$ eigenvalue of the Hilbert-Schmidt operator $\left(\frac{1}{[i, j]}\right)_{i, j \geq 1}$.

It is natural to ask what happens for $\frac{1}{4} \leq \alpha<\frac{1}{2}$. As $\mu_{1}(\alpha)=\left\|E_{\alpha}\right\|-$ the operator norm of $E_{\alpha}$ - could (4.1) remain true for all $\alpha<\frac{1}{2}$ ? This follows if we can show $E_{\alpha}$ is bounded in this range and $\left\|\left(E_{\alpha}\right)_{n}-E_{\alpha}\right\| \rightarrow 0$ as $n \rightarrow \infty$.

(b) Take $f(n)=\frac{\mu(n)}{n^{\alpha}}$, which is multiplicative, but not completely. Then $F(x) \sim \frac{x^{1-2 \alpha}}{\zeta(2)(1-2 \alpha)}$ for $\alpha<\frac{1}{2}$, which is regularly varying of index $\rho=1-2 \alpha$. Again $\rho>\frac{1}{2}$ if and only if $\alpha<\frac{1}{4}$. In this case one finds that

$$
\lambda(k)=\frac{\mu(k)}{\sigma_{-1}(k) k^{\alpha}},
$$

where $\sigma_{-1}(n)=\sum_{d \mid n} \frac{1}{d}$. The series $\sum_{n=1}^{\infty} \frac{|\lambda(n)|^{2}}{n^{2 \rho-\delta}}$ therefore converges for $\alpha<\frac{1-\delta}{2}$. Also for $(k, l)=1$ and, using $|\mu(m n)| \leq|\mu(m)||\mu(n)|$, we have

$$
\left|F_{k, l}(x)\right| \leq \sum_{n \leq x} \frac{|\mu(k n) \mu(l n)|}{(k n)^{\alpha}(l n)^{\alpha}} \leq \frac{|\mu(k l)|}{(k l)^{\alpha}} \sum_{n \leq x} \frac{|\mu(n)|^{2}}{n^{2 \alpha}}=\sigma_{-1}(k l)|\lambda(k l)| F(x) \ll_{\varepsilon}(k l)^{\varepsilon}|\lambda(k l)| F(x)
$$

for all $\varepsilon>0$, so the conditions of Theorem 3.2 are satisfied. Hence, for $\alpha<\frac{1}{4}$, the $r^{\text {th }}$ singular value of $M_{n}(f)$ is asymptotic to $\sqrt{\frac{\nu_{r}}{\zeta(2)(1-2 \alpha)}} n^{\frac{1}{2}-\alpha}$, where $\nu_{r}$ is the $r^{\text {th }}$ eigenvalue of the Hilbert-Schmidt operator

$$
G_{\alpha}=\left(\frac{\mu\left(\frac{[i, j]}{(i, j)}\right)(i j)^{\alpha}}{\sigma_{-1}\left(\frac{[i, j]}{(i, j)}\right)[i, j]}\right)_{i, j \geq 1}
$$

Again for $\alpha=0$, this was discussed in [13] and [1]. Note that $M_{n}(\mu)=M_{n}(1)^{-1}$, so $M_{n}(u)$ has singular values $\frac{1}{\lambda_{n, n}}, \cdots, \frac{1}{\lambda_{1, n}}$ (in decreasing order) where $\lambda_{1, n}, \cdots, \lambda_{n, n}$ are the singular values of $M_{n}(1)$. Mattila and Haukkanen [13] conjectured and Altinişik and Büyükköse [1] proved that $\lambda_{n, n}$ decreases with $n$, and further proved that $\lambda_{n, n}^{2} \leq \frac{1}{Q(n)} \sim \frac{\zeta(2)}{n}$, where $Q(n)=\sum_{r=1}^{n} \mu(r)^{2}$. The above (with $\alpha=0$ ) shows that in fact

$$
\frac{1}{\lambda_{n-r+1, n}^{2}} \sim \frac{\nu_{r}}{\zeta(2)} n \quad \text { as } n \rightarrow \infty \quad(r=1,2,3, \ldots)
$$

where $\nu_{r}$ is the $r^{\text {th }}$ eigenvalue of $G_{0}$.

\section{References}

[1] E. Altinişik and Ş. Büyükköse, A proof of a conjecture on monotonic behaviour of the smallest and the largest eigenvalues of a number theoretic matrix, Linear Algebra and its Applications 471 (2015) 141-149.

[2] N. H. Bingham, C. M. Goldie, and J. L. Teugels, Regular Variation, Cambridge University Press, 1987.

[3] A. Böttcher and B. Silbermann, Introduction to large truncated Toeplitz matrices, Springer-Verlag, 1999.

[4] L. A. Coburn, R. D. Moyer, and I. M. Singer, $C^{*}$-Algebras of almost periodic pseudo-differential operators, Acta Math. 130 (1973) 297-307.

[5] J. Dixmier, Les Algebras d'Operateurs dans l'Espace Hilbertien, Gunthier-Villans, 1969.

[6] N. Dunford and J. T. Schwartz, Linear Operators Part II, Wiley and Sons, 1964. 
[7] I. Gohberg, S. Gohberg, and M. A. Kaashoek, Classes of Linear Operators Vol. 1, Operator Theory Advances and Applications Vol. 49, Birkhäuser, 1990.

[8] T. W. Hilberdink, Determinants of Multiplicative Toeplitz matrices, Acta Arith. 125 (2006) 265-284.

[9] T. W. Hilberdink, An arithmetical mapping and applications to $\Omega$-results for the Riemann zeta function, Acta Arith. 139 (2009) 341-367.

[10] T. W. Hilberdink, Multiplicative Toeplitz Operators and the Riemann zeta function, in: EMS Series of Lectures in Mathematics, European Mathematical Society 2015.

[11] S. Hong and R. Loewy, Asymptotic behavior of eigenvalues of greatest common divisor matrices, Glasg. Math. J. 46 (2004) 551-569.

[12] T. J. Jarvis, A dominant negative eigenvalue of a matrix of Redheffer, Linear Algebra and its Applications 142 (1990) 141-152.

[13] M. Mattila and P. Haukkanen, On the eigenvalues of certain number-theoretic matrices, East-West J. Math. 14 (2012) 121-130.

[14] R. M. Redheffer, Eine explizit lösbare Optimierungsaufgabe, Internat. Schriftenreihe Numer. Math. 36 (1977)

\section{Appendix}

\section{Proposition A1}

Let $\left(a_{n}\right)_{n \in \mathbb{N}}$ be a non-negative sequence of reals, not identically zero, and let $A(x)=\sum_{n \leq x} a_{n}$. Then the limit

$$
\lim _{n \rightarrow \infty} \frac{A(n / k)}{A(n)}
$$

exists for every $k \in \mathbb{N}$ and is non-zero if and only if $A(\cdot)$ is regularly varying.

Proof. If $A(\cdot)$ is regularly varying, then the limit in $(*)$ exists and is of the form $k^{-\rho}$ for some $\rho \geq 0$.

For the converse, we first show that the limit in $(*)$ must be of the form $k^{-\rho}$ for some $\rho \geq 0$. For, denoting the limit by $h(k)$, we find that $h(\cdot)$ is completely multiplicative, non-negative, and decreasing.

Let $p$ be a prime number. For each $k \in \mathbb{N}, 2^{m} \leq p^{k}<2^{m+1}$ for some $m \in \mathbb{N}$. Indeed, $m=\left[\frac{k \log p}{\log 2}\right]$. Since $h$ is decreasing and completely multiplicative, we have

$$
h(2)^{m} \geq h(p)^{k} \geq h(2)^{m+1} .
$$

It follows that $m=\left[\frac{k \log h(p)}{\log h(2)}\right]+0$ or 1 , and hence

$$
\frac{\log h(p)}{\log h(2)}=\frac{\log p}{\log 2}+O\left(\frac{1}{k}\right)
$$

Letting $k \rightarrow \infty$ gives $h(p)=p^{-\rho}$, where $\rho=-\frac{\log h(2)}{\log 2}$. Hence $h(n)=n^{-\rho}$ for all $n \in \mathbb{N}$.

Next we show that $A(\cdot)$ is regularly varying of index $\rho$. For $m \in \mathbb{N}$, we have $A(x)=A\left(\frac{m x}{m}\right) \sim$ $m^{-\rho} A(m x)$, so that $A(m x) \sim m^{\rho} A(x)$. Hence, for rationals $q=\frac{m}{n}(m, n \in \mathbb{N})$, we have

$$
A(q x)=A\left(\frac{m}{n} x\right) \sim m^{\rho} A\left(\frac{x}{n}\right) \sim\left(\frac{m}{n}\right)^{\rho} A(x)=q^{\rho} A(x) .
$$

Finally, for $\lambda>0$, there exist sequences $\left(p_{n}\right),\left(q_{n}\right)$ of rationals such that $p_{n} \nearrow \lambda$ and $q_{n} \searrow \lambda$. As $A(\cdot)$ is increasing, we have

$$
\frac{A(\lambda x)}{A(x)} \geq \frac{A\left(p_{n} x\right)}{A(x)} \rightarrow p_{n}^{\rho}
$$


and

$$
\frac{A(\lambda x)}{A(x)} \leq \frac{A\left(q_{n} x\right)}{A(x)} \rightarrow q_{n}^{\rho} .
$$

These are true for all $n$, so that $A(\lambda x) \sim \lambda^{\rho} A(x)$ follows, and $A(\cdot)$ is regularly varying. 